\title{
Reply to the letter by Law et al. Is Rituximab therapy in Castleman's disease a risk factor for Kaposi sarcoma reactivation?
}

\author{
Paolo Nicoli · Angelo Guerrasio
}

Received: 7 January 2010/Accepted: 7 January 2010/Published online: 10 February 2010

(C) The Japanese Society of Hematology 2010

The unusual case reported by Law $\mathrm{AB}$ and co. underlines the complex (and largely unknown) interplay occurring between HHV8 infection, MCD, KS and Rituximab treatment [1]. The clinical course of the patient is more similar to what we usually observe in MCD HIV-positive patients treated with Rituximab than in MCD HIV-negative cases. Of the nine HIV-negative MCD cases treated with Rituximab-based chemotherapy or Rituximab-alone reported in literature, only one (the present case) showed KS reactivation with an abrupt onset and an aggressive clinical course. However, the occurrence of such event is not entirely surprising considering that both HHV8-positive MCDs and KS, outside of HIV-positive cohort, mostly occur in elderly people believed to have some "criptic" form of immunosuppression. In this context, the B cell depletion induced by Rituximab could represent a doublededged sword: the benefit obtained by inducing MCD remission may be offset by a reduced immunosurveillance against cancer or other infectious agents.

A body of data suggests indeed that B cell, apart from antibody production, have important immunoregulatory functions on $\mathrm{T}$ cell subsets as well act as antigen presenting cells [2].

On the other hand, the efficacy of Rituximab in nonhematological conditions like rheumatologic and renal diseases and solid organ transplantation suggest a mechanism of action not only mediated by antibody suppression.

This author's reply refers to the letter to the editor at doi:10.1007/s12185-010-0497-9.

P. Nicoli · A. Guerrasio $(\square)$

Department of Clinical and Biological Sciences,

University of Turin, San Luigi Hospital,

Regione Gonzole 10, 10043 Orbassano, Turin, Italy

e-mail: angelo.guerrasio@unito.it
Moreover, some reports have described the occurrence or an unusual accelerated growth of solid tumors during Rituximab treatment for lymphoid disorders [3, 4].

In conclusion, the efficacy of Rituximab-based therapies (we suggest Rituximab alone) in HIV-negative MCD seems undoubted; we agree with Law and co. that a strict vigilance of patients after treatment is advisable in order to monitor short and long-term effects of therapy. Last, continuing to report patients with MCD may help to better define the clinical profile and outcome of this rare disorder.

\section{References}

1. Nicoli P, Familiari U, Bosa M, Allice T, Mete F, Morotti A, et al. HHV8-positive, HIV-negative multicentric Castleman's disease: early and sustained complete remission with rituximab therapy without reactivation of Kaposi sarcoma. Int J Hematol. 2009; 90(3):392-6.

2. von Bergwelt-Baildon MS, Vonderheide RH, Maecker B, Hirano N, Anderson KS, Butler MO, et al. Human primary and memory cytotoxic $\mathrm{T}$ lymphocyte responses are efficiently induced by means of CD40-activated B cells as antigen-presenting cells: potential for clinical application. Blood. 2002;99(9):3319-25.

3. Fogarty GB, Bayne M, Bedford P, Bond R, Kannourakis G. Three cases of activation of cutaneous squamous-cell carcinoma during treatment with prolonged administration of rituximab. Clin Oncol (R Coll Radiol). 2006;18(2):155-6.

4. Wirges ML, Saporito F, Smith J. Rapid growth of Merkel cell carcinoma after treatment with rituximab. J Drugs Dermatol. 2006; 5(2):180-1. 